\title{
Eine Alternative: die beruflichen Abklärungsstellen (BEFAS)
}

\section{Klaus Jahn}

Dr. med., selbständiger Psychiater mit eigener Praxis und BEFAS-Psychiater im Appisberg, Männedorf

\author{
Die Diskussion über die gutachterliche Aussagekraft der MEDAS-Gutachten an- \\ gesichts der Abhängigkeit der MEDAS-Stellen von praktisch einem Auftraggeber \\ (der Invalidenversicherung) wird leidenschaftlich geführt. Vor diesem Hinter- \\ grund soll die Arbeit der BEFAS-Stellen der Ärzteschaft bekannter gemacht und als \\ mögliche Alternative in einer Anzahl von komplexen Fällen aufgezeigt werden.
}

\section{Summary}

BEFAS - une alternative à l'expertise médico-théorique en cas d'incapacité de travail

L'évaluation par des centres d'observation professionnelle se rapportant au jugement de l'aptitude au travail des personnes ayant des problèmes de santé complexes représente une alternative importante par rapport à la seule expertise médicale. Contrairement à celle-ci, elle offre la chance de pouvoir répondre plus objectivement aux multiples questions qui se posent autour de l'aptitude au travail, et ceci au moyen de situations de travail simulées et par la durée de l'évaluation de quatre semaines qui résultent à un grand nombre d'observations et qui permettent un jugement plus objectif de la capacité de travail. En plus, I'orientation professionnelle et l'élaboration de perspectives de travail avec les clients engendrent dans beaucoup de cas un effet encourageant et motivant et aident les clients à vaincre des tendances à la résignation et de retrouver le chemin vers un retour au marché de l'emploi.

\section{Was ist die BEFAS?}

Die BEruFlichen AbklärungsStellen (BEFAS) wurden 1982 vom Bundesamt für Sozialversicherungen ins Leben gerufen und dienen der Abklärung der praktischen Verwendung der vorhandenen Arbeitsfähigkeit von Personen, die sich selbst als arbeitsunfähig erklären und meistens eine Rente beanspruchen. Aus Sicht der IV erscheint jedoch eine (Wieder-)Eingliederung in der freien Wirtschaft durchführbar, weil sie nicht in erheblichem Masse gesundheitlich beeinträchtigt sind. Die BEFAS-Abklärung stellt eine in der Regel einmonatige funktionelle Evaluation der Ressourcen von Klienten mit gesundheitlichen (Mehrfach-)Einschränkungen dar und kann von der Invalidenver- sicherung oder anderen Versicherungen in Auftrag gegeben werden.

Sie schliesst eine eingehende Berufsberatung durch erfahrene Berufs- und Laufbahnberater sowie eine medizinische Beurteilung ein und hat gegenüber der konventionellen medizinisch-theoretischen Begutachtung eine Reihe von Vorteilen, da die Klienten in einem der Arbeitswelt nachempfundenen Rahmen an verschiedenen simulierten Arbeitsplätzen über einen Monat eingesetzt und evaluiert werden können. So lässt sich häufig eine validere Einschätzung der Leistungsfähigkeit und der beruflichen Einsatzmöglichkeiten eines Klienten erarbeiten, da sich viele Störungen in diesem Rahmen alltagsnaher beurteilen lassen.

Die BEFAS-Ärzte haben den Vorteil, die medizinisch-theoretischen Resultate der vorgängigen ärztlichen Abklärungen zum einen auf ihre Plausibilität und Vollständigkeit hin überprüfen, aber auch eine genauere Einschätzung der Arbeits- und Leistungsfähigkeit vornehmen zu können. Für die psychiatrischen Abklärungen ist hier die Verhaltensbeobachtung während des Abklärungsprozesses besonders aufschlussreich. Insbesondere Phänomene wie Selbstlimitierung und Aggravation sind hier leichter als in der konventionellen Begutachtenssituation beurteilbar, da es schwierig ist, diese über den ganzen Abklärungsverlauf hin konsistent und widerspruchsfrei zu produzieren.

Durch die Betonung und Ermittlung der Ressourcen und das Aufzeigen von Möglichkeiten trotz der gesundheitlichen Einschränkungen gelingt es häufig, Klienten mit einer resignativen Grundhaltung für einen Weg zurück in den ersten Arbeitsmarkt zu moti- 


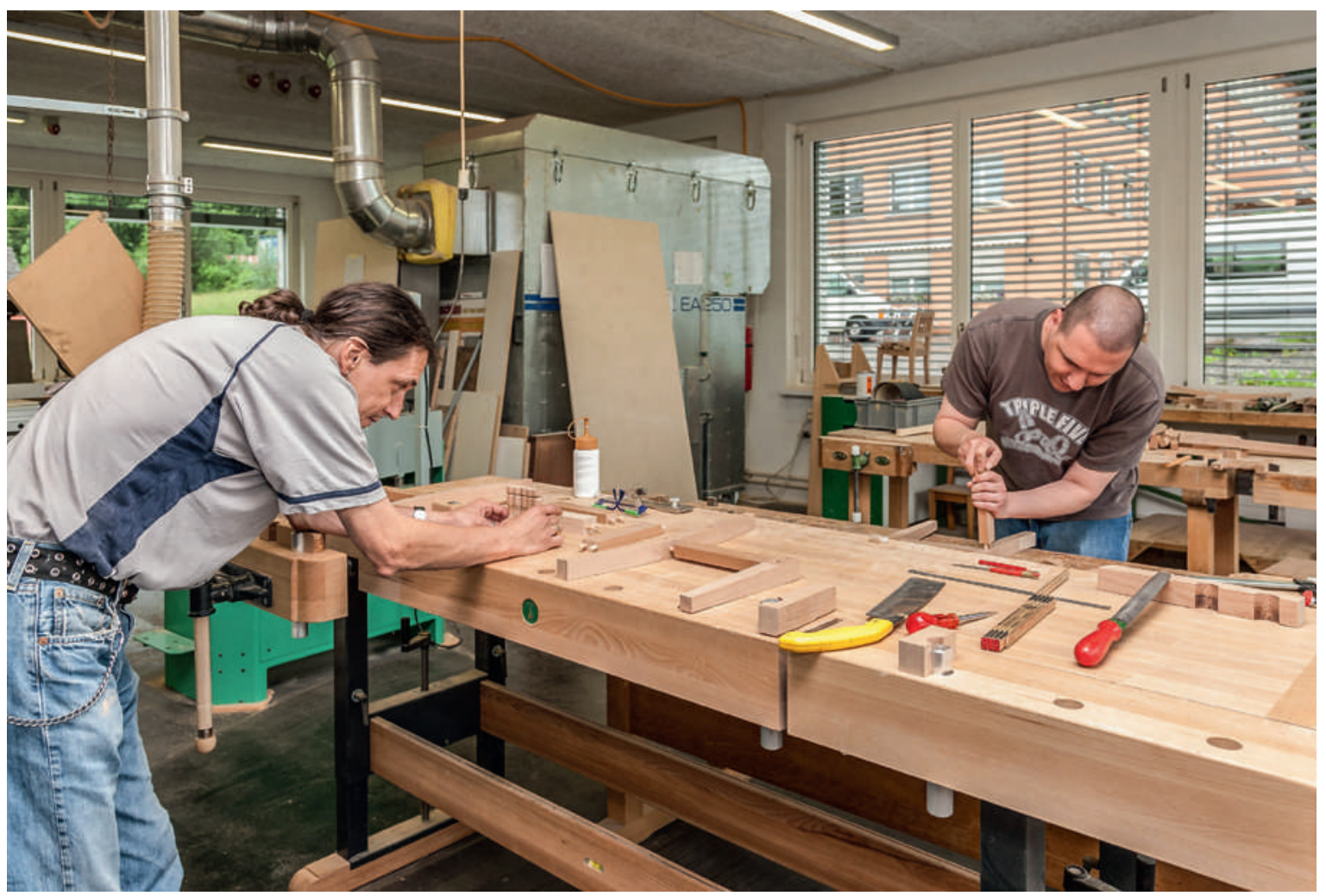

Erfahrungen von einem Monat berufspraktischer Abklärung mit Einsatz an vier verschiedenen Orten fliessen in die BEFAS-Beurteilung ein.

vieren, die ohne die BEFAS-Abklärung den Weg des Rentenbegehrens eingeschlagen hätten.

\section{Wer profitiert von einer BEFAS-Abklärung?}

Versicherte mit gesundheitlicher Mehrfachproblematik, die sich selbst nicht oder nur teilweise als arbeitsund eingliederungsfähig erleben, weil sie in der Bewältigung ihrer gesundheitlichen Einschränkungen und mit ihrer Wiedereingliederung überfordert sind und/oder Rentenleistungen beanspruchen. Besonders geeignet ist die BEFAS-Abklärung für Jugendliche mit abgebrochener Erstausbildung, aber auch für ältere Arbeitnehmer, die z.B. unfallbedingt eine komplette berufliche Neuorientierung vornehmen müssen.

- Interdisziplinär im Team:

Allgemeinmediziner, Psychiater, Berufsberater/in + Arbeitsabklärer/in
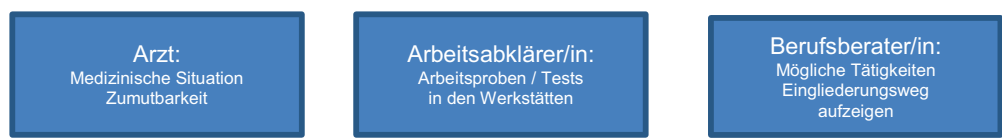

- Mitwirkungsmöglichkeiten aufzeigen

\section{- realistische Anschlusslösungen}

Zusammensetzung und Aufgaben eines BEFAS-Teams.
Die BEFAS Appisberg konnte 2013 in den 193 Abklärungen folgende Empfehlungen abgeben:

\begin{tabular}{lcc}
\hline Empfehlungen 2013 & Anzahl & Anteil \% \\
\hline Arbeitstraining in freier Wirtschaft & 13 & 7 \\
\hline Arbeitstraining im geschützten Rahmen & 42 & 22 \\
\hline Einarbeitung/Stellenvermittlung & 58 & 30 \\
\hline Umschulung in freier Wirtschaft & 43 & 22 \\
\hline Umschulung im geschützten Rahmen & 15 & 8 \\
\hline Angestammter Beruf, & 6 & 3 \\
bisheriger Arbeitgeber & 14 & 7 \\
\hline Geschützter Arbeitsplatz & 2 & 1 \\
\hline Weiterführende Schulen & 193 & 100 \\
\hline Gesamt/Total & & \\
\hline Eingliederung mit besonderer & 57 & 30 \\
\hline Unterstützung im geschützten Rahmen & & \\
\hline Eingliederung direkt in der freien & 122 & 63 \\
Wirtschaft & 14 \\
\hline Keine Eingliederung möglich (Rente) & 7 \\
\hline
\end{tabular}

Dies bestätigt, dass zwei Drittel mit Unterstützung durch die IV direkt in die freie Wirtschaft eingegliedert werden können, während ein knapper Drittel besonderer Unterstützung für eine Wieder-Eingliederung bedarf. Nur in $7 \%$ der Fälle ist eine Rentenzusprache unumgänglich. 


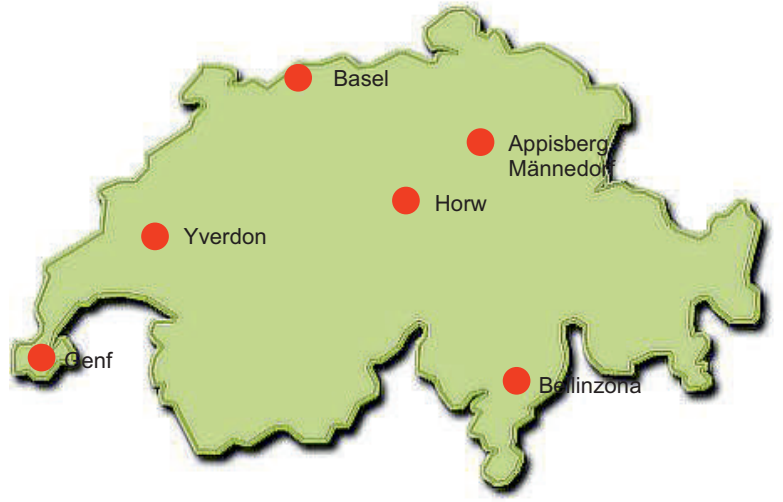

Die Standorte der beruflichen Abklärungsstellen BEFAS in der Schweiz.

Diese Zahlen unterlagen in den letzten Jahren nur kleinen Schwankungen und waren in allen BEFASStellen ähnlich.

\section{Was sind die wichtigsten Unterschiede zwischen einer BEFAS- und einer MEDAS- Begutachtung?}

Eine MEDAS-Begutachtung soll die medizinische Situation durch eine gründliche ärztlich-gutachterliche Untersuchung aller involvierten Fachrichtungen objektivieren und den Leistungserbringer in die Lage versetzen, seinen Entscheid über berufliche Massnahmen oder eine Rentenleistung auf einer soliden Entscheidungsbasis fällen zu können.

Fragen der möglichen Leistungs- und Eingliederungsfähigkeit werden aufgrund medizin-theoretischer Überlegungen beantwortet und nehmen in der Exploration und Untersuchung der Klienten vergleichsweise wenig Raum ein. Die Klienten erleben die MEDAS-Begutachtung in der Regel als sehr umfangreich und gründlich, aber auch als distanziert und teilweise feindlich "wie bei einem Verhör». Zum Zeitpunkt einer MEDAS-Begutachtung sind oft schon Rechtsvertreter involviert und eine Verhärtung auf beiden Seiten ist spürbar, da die Einschätzungen von Seiten der Versicherten und der IV-Stelle stark divergieren.

Diese Aspekte sind auch bei den BEFAS-Abklärungen spürbar, wiegen aber weniger schwer, da der Fokus auf den verbleibenden Ressourcen und deren beruflicher Verwertbarkeit liegt. Durch das Erleben von Ressourcen und die Klärung beruflicher Perspektiven gelingt es im Idealfall, die Resignation und Angst der Versicherten zu durchbrechen und sie neu zu motivieren. Auch gelingt es meistens, Fälle von Selbstlimitation oder Aggravation zu benennen und durch eine genaue Beschreibung des Verhaltens argumentativ zu untermauern, so dass die IV-Stelle sich darauf berufen und etwaige unbegründete Begehren abweisen kann.

\section{Wo liegen die Grenzen einer BEFAS-Abklärung?}

Wie auch bei der medizinisch-theoretischen Begutachtung sind Einschätzungen von Arbeits- und Leistungsfähigkeit durch die subjektive Sichtweise des Untersuchers beeinflusst und nicht vollständig objektiv.

Im Gegensatz zur medizinisch-theoretischen Begutachtung haben die Berufsabklärer und Ärzte der BEFAS die Möglichkeit, die Erfahrungen von einem Monat berufspraktischer Abklärung mit Einsatz an vier verschiedenen Orten und einer genauen Verhaltensbeobachtung in die Beurteilung einfliessen zu lassen.

Die finale Beurteilung wird im Team im Konsens erarbeitet und weist somit z.B. bei psychiatrischen $\mathrm{Pa}$ tienten ein viel grösseres Erfahrungsspektrum auf. Meistens umfasst dies auch Beobachtungen, die sich in der konventionellen Begutachtungssituation nicht zeigen und nicht oder nur schwer fassen liessen.

Eine geringe Anzahl von Klienten hat Mühe, sich auf das BEFAS-Setting einzulassen. Häufig liegen hier narzisstische Akzentuierungen der Persönlichkeit oder ausgeprägte Unreife vor und die Klienten erleben z.B. einen Einsatz in einer Holzwerkstatt als unter ihrer Würde oder sind durch die Realitätskonfrontation hinsichtlich ihres realistischen beruflichen Potentials gekränkt. Aber auch diese, für beide Seiten

\section{Der Fokus liegt auf den verbleibenden} Ressourcen und deren beruflicher Verwertbarkeit.

schwierigen, Abklärungen führen zu wichtigen Erkenntnissen, denn diese Probleme würden sich auch am Arbeitsplatz bemerkbar machen.

\section{Fazit}

Die geringen Mehrkosten der BEFAS-Abklärung gegenüber einer interdisziplinären MEDAS-Begutachtung relativieren sich rasch, wenn bei einer Anzahl von Klienten eine rentenausschliessende Reintegration in den Arbeitsmarkt gelingt oder auch nur ein langwieriger und zermürbender Rechtsstreit vermieden werden kann, indem mit dem Versicherten und der zuständigen IV-Stelle eine gemeinsame Sichtweise erarbeitet werden kann. 